at the base for steady evaporation. The rate of evaporation can be controlled by adjusting this opening. During the course of the next few hours, when no attention is necessary, part of the mixture travels farther up, forming a distinct zone (see Fig. 1). It is important that this process should be stopped at this stage, otherwise the lower zone also will rise and the separation obtained earlier is lost. The filter paper is cut in between the two zones and each run separately until pure fractions are obtained.

Using the above method, some known mixtures, (1) phenanthrene and anthracene, (2) naphthalene and bi-phenyl, (3) $\alpha$-naphthol and $\beta$-naphthol, (4) hydroquinone and resorcinol, were easily separated. Hydroquinone and resorcinol, however, crystallized so well within the pores of the filter paper that one could not scrape them off without tearing the filter paper. Using wider strips, it is easy to separate larger quantities of substances in a short time.

Indian Institute of Science,

R. C. VASISTH

M. S. MUTHana

Bangalore, and

Indian Institute of Technology, Kharagpur. July 22.

1 Schonbein, Ann., 114, 275 (1861).

2 Goppelsroeder, Monograph, "Capillar und Adsorptions Analyse" in J Houben, "Die Methoden der Organischen Chemie", 1, 291 (Leipzig, 1925).

\section{Phenylation, Benzoyloxylation and Halogenation of Aromatic Compounds by Silver Benzoate - Halogen Complexes}

Atthough the reaction between halogens and the silver salts of carboxylic acids constitutes a fairly well-known method for the preparation of organic halides, its mechanism is far from clear. Several types of intermediate have been isolated or postulated by different workers ${ }^{1,2}$. Simonini ${ }^{3}$ obtained complexes which he formulated as $\left(R \mathrm{CO}_{2}\right)_{2} \mathrm{AgX}$ (where $X=$ halogen), for example, the so-called 'silver iodide dibenzoate'. Birckenbach and Meisenheimer examined the decomposition of certain of these complexes in the presence of benzene, using a reaction between one equivalent of the silver salt and two equivalents of iodine. The formation of iodobenzene and the phenyl ester of the acid was reported. From a reaction between silver $p$-chlorobenzoate and iodine in boiling chlorobenzene, $p$-chlorophenyl $p$-chlorobenzoate was isolated. In this latter case, it is not clear from the experimental evidence whether the ester resulted from a reaction with the solvent, or whether both its $p$-chlorophenyl groups originated in the silver salt. It is known that esters, $R \mathrm{CO}_{2} R$, can result from analogous reactions between silver salts, $R \mathrm{CO}_{2} \mathrm{Ag}$, and iodine $\theta^{5}$. A preliminary investigation of this sub. ject has exposed several interesting new features, which are now briefly described.

Silver benzoate has been suspended in a number of aromatic compounds and treated with one equiva. lent of bromine or iodine at $20^{\circ}$. The resulting insoluble complex has been caused to decompose by heating at $80^{\circ}(\mathrm{Br})$ or $130^{\circ}(\mathrm{I})$. Silver iodide dibenzoate decomposes in chlorobenzene at $130^{\circ}$ with the evolution of carbon dioxide and the formation of a mixiure of isomeric chlorodiphenyls ( 22 per cent), chlorophenyl benzoates ( 5 per cent) and benzoic acid
(35 per cent); the iodine appears almost quantitatively as silver iodide. Reduction of the chlorodiphenyls with Raney nickel gave diphenyl, and bromination gave a little 4-bromo-4'-chlorodiphenyl, melting point and mixed melting point $154-155 \cdot 5^{\circ}$. The relative proportions of phenylation and benzoyloxylation in this experiment are similar to those which are found in the decomposition of benzoyl peroxide in aromatic solvents ${ }^{6}$, and suggest a common basic mechanism, namely, the formation and sub. sequent decarboxylation of free benzoyloxyl radicals, $\mathrm{C}_{6} \mathrm{H}_{5} \mathrm{CO}_{2}$.

In contrast, the decomposition of silver bromide dibenzoate in chlorobenzene leads to benzoyloxylation only, and not to any appreciable degree of phenylation. Of the mixture of chlorophenyl benzoates which was obtained ( 23 per cent), 30 per cent of the para isomer was readily isolated by erystallization : the true proportion was certainly higher than this. Only 70 per cent of the bromine appears as silver bromide. In nitrobenzene, silver bromide dibenzoate decomposes to give a mixture of nitrodiphenyls and $m$-nitrophenyl benzoate in approximately equal proportions. Little, if any, of the para ester is produced, for a blank experiment showed that the working-up process would tend to concentrate this isomer. The orientation in these benzoyloxylation experiments is regarded as a significant indication of their electrophilic character and of the intermediacy of benzoyloxyl cations-a hitherto unknown species. Unfortunately, the orientation in the homolytic benzoyloxylation of benzene derivatives is not yet known, and direct comparison with the present results is not possible. In homolytic phenylation and hydroxylation, meta positions are known to be relatively unreactive, and the effect of substituents in the aromatic ring is small. A homolytic mechanism for benzoyloxylation by silver bromide dibenzoate is therefore regarded as improbable. It may be noted in passing that this reaction represents a method for the direct oxidation of the aromatic nucleus.

Another type of reaction is illustrated by the decomposition of silver bromide dibenzoate (prepared in $n$-heptane) in anisole. This gives $p$-bromoanisole as the principal high-boiling product; but slight benzoyloxylation of the anisole also occurs. Silver iodide dibenzoate and silver iodide diacetate oxidize isopropylbenzene to acetophenone at $100^{\circ}$; the yields are low.

It is already clear that a common mechanism cannot be advanced for all reactions which involve halogens and the silver salts of carboxylic acids ; but it is felt that detailed considerations should await the results of further experiments. An account of these will be given elsewhere in due course.

I would like to acknowledge the interest shown in this work by Prof. D. H. Hey and Dr. G. H. Williams. The work is being carried out during the tenure of an I.C.I. Research Fellowship at the University of London.

Chemistry Department,

\section{Bryce-Smith}

King's College,

London, W.C.2. July 17.

${ }^{1}$ Kleinberg, Chem. Rev., 40, 381 (1947).

'Johnson, Ann. Rep. Chem. Soc., 46, 154 (1949).

3 Simonini, Monatsh., 14, 81 (1893).

4 Birckenbach and Meisenheimer, Ber., 69, 723 (1936).

- Wieland and Fischer, Ann., 446, 49 (1926).

- Cadogan, Hey and Williams (private communication). 